\title{
ON SPECIAL VALUES OF STANDARD $L$-FUNCTIONS ATTACHED TO VECTOR VALUED SIEGEL MODULAR FORMS
}

\author{
NoRITOMO KOZIMA
}

\section{Introduction}

Let $V$ be a vector space of dimension $n \in Z_{>0}$ over $C$ and $\operatorname{sym}^{l}(V)$ the $l$-th symmetric tensor product of $V$ with $l \in Z_{\geq 0}$. For $k \in Z_{\geq 0}$, let $f$ be a $\operatorname{sym}^{l}(V)$ valued Siegel modular form of type $\operatorname{det}^{k} \otimes \operatorname{sym}^{l}$ with respect to $S p(n, Z)$ (size $2 n$ ). Suppose $f$ is a cuspform and an eigenform (i.e., a non-zero common eigenfunction of the Hecke algebra). Then we define the standard $L$-function attached to $f$ by

$$
L(s, f, \underline{\mathrm{St}}):=\prod_{p}\left\{\left(1-p^{-s}\right) \prod_{j=1}^{n}\left(1-\alpha_{j}(p) p^{-s}\right)\left(1-\alpha_{j}(p)^{-1} p^{-s}\right)\right\}^{-1},
$$

where $p$ runs over all prime numbers and $\alpha_{j}(p)(j=1, \ldots, n)$ are the Satake $p$ parameters of $f$. The right-hand side of (1.1) converges absolutely and locally uniformly for $\operatorname{Re}(s)>n+1$. We put

$$
\Lambda(s, f, \underline{\mathrm{St}}):=\Gamma_{R}(s+\varepsilon) \Gamma_{C}(s+k+l-1) \prod_{J=2}^{n} \Gamma_{C}(s+k-j) L(s, f, \underline{\mathrm{St}})
$$

with

$$
\Gamma_{\boldsymbol{R}}(s):=\pi^{-s / 2} \Gamma\left(\frac{s}{2}\right), \quad \Gamma_{C}(s):=2(2 \pi)^{-s} \Gamma(s)
$$

and

$$
\varepsilon:= \begin{cases}0 & \text { for } n \text { even } \\ 1 & \text { for } n \text { odd }\end{cases}
$$

Then by Takayanagi [9, Theorem 2, Theorem 3], we have:

If $k, l \in 2 Z, k>0, l \geq 0$, then $\Lambda(s, f, \underline{\mathrm{St}})$ has a meromorphic continuation to the whole s-plane and satisfies the functional equation

$$
\Lambda(s, f, \underline{\mathrm{St}})=\Lambda(1-s, f, \underline{\mathrm{St}})
$$

Recelved May 14, 1999. 
and if $k>n$, then $\Lambda(s, f, \underline{\mathrm{St}})$ is holomorphic except for possible simple poles at $s=0$ and $s=1$. Moreover if $n \not \equiv 0(\bmod 4)$, then $\Lambda(s, f, \underline{\mathrm{St}})$ is entire.

Therefore the right half of critical points of $L(s, f, \underline{\mathrm{St}})$ is

$$
\{m \in \boldsymbol{Z} \mid 1 \leq m \leq k-n \text { and } m \equiv n(\bmod 2)\} .
$$

For scalar valued cases (i.e., $l=0)$, special values of $L(s, f, \underline{\mathrm{St}})$ were studied by several authors: Sturm [8], Harris [5], Böcherer [2], and Mizumoto [6]. In this paper, we give some algebraic results for the values in the case of vector valued modular forms. The main theorem is follows:

THEOREM (Precise statements are given below). Let $k, l \in 2 \boldsymbol{Z}_{\geq 0}$ and $k \geq 2 n+2$. Let $f$ be a $\operatorname{sym}^{l}(V)$-valued cuspidal eigenform of type $\operatorname{det}^{k} \otimes \operatorname{sym}^{l}$. Let $Q(f)$ be the extension field of $\boldsymbol{Q}$ generated by the eigenvalues on $f$ of the Hecke algebra over $\boldsymbol{Q}$. Suppose the Fourier coefficients of $f$ in $\boldsymbol{Q}(f)$.

Let $m \in Z$ be in the right half of critical points of $L(s, f, \underline{\mathrm{St}})$. If $m=1$, then we assume $n \equiv 3(\bmod 4)$. Let

$$
A(f):=\frac{L(m, f, \underline{\mathrm{St}})}{\pi^{n k+l+m(n+1)-n(n+1) / 2}(f, f)} .
$$

Then we have

$$
A(f)^{\sigma}=A\left(f^{\sigma}\right) \quad \text { for all } \sigma \in \operatorname{Aut}(C)
$$

In particular,

$$
A(f) \in \boldsymbol{Q}(f)
$$

\section{Preliminaries} [10]).

In this section, we describe notations and basic notions (see [3], [6], [9] and

Let $n \in \boldsymbol{Z}_{>0}, k, l \in \boldsymbol{Z}_{\geq 0}$. Let $x=\left(x_{1}, \ldots, x_{n}\right)$ be a row vector consisting of $n$ indeterminates. We put

$$
V:=C x_{1} \oplus \cdots \oplus \boldsymbol{C} x_{n}
$$

and define a Hermitian inner product on $V$ by

$$
\left\langle\sum_{j=1}^{n} a_{j} x_{j}, \sum_{j=1}^{n} b_{j} x_{j}\right\rangle:=\sum_{j=1}^{n} a_{j} \overline{b_{j}}
$$

where $a_{j}, b_{j} \in \boldsymbol{C}(j=1, \ldots, n)$.

We identify $\operatorname{sym}^{l}(V)$ with the $C$-vector space of homogeneous polynomials in $x$ of degree $l$. The inner product on $V$ induces an inner product on $\operatorname{sym}^{l}(V)$ defined by 


$$
\left\langle v_{1} \cdots v_{l}, w_{1} \cdots w_{l}\right\rangle:=\frac{1}{l !} \sum_{\tau \in \mathfrak{S}_{l}} \prod_{j=1}^{l}\left\langle v_{\tau(j)}, w_{j}\right\rangle,
$$

where $v_{j}, w_{j} \in V(j=1, \ldots, l)$.

Let $\rho$ be the representation of $G L(n, C)$ on $\operatorname{sym}^{l}(V)$ defined by

$$
\rho(g)(v(x))=(\operatorname{det} g)^{k} v(x g), \quad v(x) \in \operatorname{sym}^{l}(V) .
$$

Let $\Gamma^{n}:=S p(n, Z)$ be the Siegel modular group of degree $n$, and $\mathfrak{H}_{n}$ be the Siegel upper half space of degree $n$. For $M=\left(\begin{array}{cc}A & B \\ C & D\end{array}\right) \in \Gamma^{n}$ and $Z \in \mathfrak{H}_{n}$, we put

$$
M\langle Z\rangle:=(A Z+B)(C Z+D)^{-1}, \quad j(M, Z):=\operatorname{det}(C Z+D),
$$

and for $f: \mathfrak{H}_{n} \rightarrow \operatorname{sym}^{l}(V)$,

$$
(f \mid M)(Z):=\rho\left((C Z+D)^{-1}\right) f(M\langle Z\rangle) .
$$

A $C^{\infty}$-function $f: \mathfrak{H}_{n} \rightarrow \operatorname{sym}^{l}(V)$ is called a $\operatorname{sym}^{l}(V)$-valued $C^{\infty}$-modular form of type $\rho$ if it satisfies $f \mid M=f$ for all $M \in \Gamma^{n}$. The space of all such functions is denoted by $M_{k, l}^{n}\left(\operatorname{sym}^{l}(V)\right)^{\infty}$. The space of $\operatorname{sym}^{l}(V)$-valued Siegel modular forms of type $\rho$ is defined by

$$
\begin{aligned}
& M_{k, l}^{n}\left(\operatorname{sym}^{l}(V)\right) \\
& \quad:=\left\{f \in M_{k, l}^{n}\left(\operatorname{sym}^{l}(V)\right)^{\infty} \mid f \text { is holomorphic on } \mathfrak{H}_{n} \text { (and its cusps) }\right\},
\end{aligned}
$$

and the space of cuspforms by

$$
\begin{aligned}
& S_{k, l}^{n}\left(\operatorname{sym}^{l}(V)\right) \\
& \quad:=\left\{f \in M_{k, l}^{n}\left(\operatorname{sym}^{l}(V)\right) \mid \lim _{\lambda \rightarrow \infty} f\left(\begin{array}{cc}
Z & 0 \\
0 & i \lambda
\end{array}\right)=0 \text { for all } Z \in \mathfrak{H}_{n-1}\right\} .
\end{aligned}
$$

For $f, g \in M_{k, l}^{n}\left(\operatorname{sym}^{l}(V)\right)^{\infty}$, the Petersson inner product of $f$ and $g$ is defined by

$$
(f, g):=\int_{\Gamma^{n} \backslash \mathfrak{H}_{n}}\langle\rho(\sqrt{\operatorname{Im} Z}) f(Z), \rho(\sqrt{\operatorname{Im} Z}) g(Z)\rangle \operatorname{det}(\operatorname{Im} Z)^{-n-1} d Z
$$

if the right-hand side is convergent.

For $f \in M_{k, l}^{n}\left(\operatorname{sym}^{l}(V)\right), f$ has a Fourier expansion of the following type:

$$
f(Z)=\sum_{R \geq 0} a_{R}(f) e^{2 \pi i \operatorname{trace}(R Z)}, \quad\left(a_{R}(f) \in \operatorname{sym}^{l}(V), Z \in \mathfrak{H}_{n}\right)
$$

where $R$ runs through symmetric, semi-integral, semi-positive matrices of size $n$, we denote such $R$ by " $R \geq 0$ ". 
Let $K$ be any subfield of $C$. We put

$$
\begin{gathered}
V_{K}:=K x_{1} \oplus \cdots \oplus K x_{n}, \\
M_{k, l}^{n}\left(\operatorname{sym}^{l}(V)\right)_{K}:=\left\{f \in M_{k, l}^{n}\left(\operatorname{sym}^{l}(V)\right) \mid a_{R}(f) \in \operatorname{sym}^{l}\left(V_{K}\right) \text { for all } R \geq 0\right\},
\end{gathered}
$$

and for any subset $X$ of $M_{k, l}^{n}\left(\operatorname{sym}^{l}(V)\right)$,

$$
X_{K}:=X \cap M_{k, l}^{n}\left(\operatorname{sym}^{l}(V)\right)_{K} .
$$

For $\sigma \in \operatorname{Aut}(\boldsymbol{C})$, we put

$$
f^{\sigma}(Z):=\sum_{R \geq 0} a_{R}(f)^{\sigma} e^{2 \pi i \operatorname{trace}(R Z)} .
$$

Then by Takei [10], if $k \geq 2 n+2$ then $S_{k, l}^{n}\left(\operatorname{sym}^{l}(V)\right)=S_{k, l}^{n}\left(\operatorname{sym}^{l}(V)\right)_{Q} \otimes_{Q} C$. Therefore $\operatorname{Aut}(\boldsymbol{C})$ acts on $S_{k, l}^{n}\left(\operatorname{sym}^{l}(V)\right)$ by $f \mapsto f^{\sigma}$.

Let $L_{C}^{(n)}\left(\right.$ resp. $\left.L_{Q}^{(n)}\right)$ be the abstract Hecke algebra of degree $n$ over $C$ (resp. $Q)$, and let

$$
t: L_{C}^{(n)} \rightarrow \operatorname{End}_{C}\left(S_{k, l}^{n}\left(\operatorname{sym}^{l}(V)\right)\right)
$$

be the $C$-algebra homomorphism defined as in [1]. We put $\mathbf{T}_{C}:=t\left(L_{C}^{(n)}\right)$ and $\mathbf{T}_{\boldsymbol{Q}}:=t\left(L_{\boldsymbol{Q}}^{(n)}\right)$.

Let $f \in S_{k, l}^{n}\left(\operatorname{sym}^{l}(V)\right)$ be an eigenform, and for $T \in \mathbf{T}_{C}$, let $\lambda(T) \in C$ be an eigenvalue on $f$ :

$$
T f=\lambda(T) f \quad \text { for all } T \in \mathbf{T}_{C} .
$$

Then $\lambda$ defines an element of

$$
\widehat{\mathbf{T}_{C}}:=\left\{\mathbf{T}_{\boldsymbol{C}} \rightarrow \boldsymbol{C}: \boldsymbol{C} \text {-algebra homomorphisms }\right\},
$$

and each element of $\widehat{\mathbf{T}_{C}}$ is obtained in this way.

For $\lambda \in \widehat{\mathbf{T}_{C}}$, we put

$$
S_{k, l}^{n}(\lambda):=\left\{f \in S_{k, l}^{n}\left(\operatorname{sym}^{l}(V)\right) \mid T f=\lambda(T) f \text { for all } T \in \mathbf{T}_{C}\right\} .
$$

Then the space of cuspforms decomposes into eigenspaces:

$$
S_{k, l}^{n}\left(\operatorname{sym}^{l}(V)\right)=\underset{\lambda \in \widehat{\mathbf{T}_{C}}}{\bigoplus_{k, l}} S_{k}^{n}(\lambda) .
$$

We note that for any $f_{J} \in S_{k, l}^{n}\left(\lambda_{J}\right)(j=1,2),\left(f_{1}, f_{2}\right)=0$ if $\lambda_{1} \neq \lambda_{2}$.

For $\lambda \in \widehat{\mathbf{T}_{\boldsymbol{C}}}$, we define an extension field of $\boldsymbol{Q}$ by

$$
\boldsymbol{Q}(\lambda):=\boldsymbol{Q}\left(\lambda(T) \mid T \in \mathbf{T}_{\mathbf{Q}}\right)
$$

and for $f \in S_{k, l}^{n}(\lambda)$, we put $\boldsymbol{Q}(f):=\boldsymbol{Q}(\lambda)$. Then by Takei [10], $\boldsymbol{Q}(\lambda)$ is a totally real finite extension of $\boldsymbol{Q}$. 


\section{Differential operator and the pullback formula}

We put

$$
\begin{aligned}
& V_{1}:=\boldsymbol{C} x_{1} \oplus \cdots \oplus \boldsymbol{C} x_{n}, \quad e_{1}:=\left(x_{1}, \ldots, x_{n}\right), \\
& V_{2}:=\boldsymbol{C} x_{n+1} \oplus \cdots \oplus \boldsymbol{C} x_{2 n}, \quad e_{2}:=\left(x_{n+1}, \ldots, x_{2 n}\right) .
\end{aligned}
$$

Let $l$ be an isomorphism from $V_{1}$ to $V_{2}$ defined by $l\left(x_{J}\right)=x_{n+\jmath}(j=1, \ldots, n)$. It induces an isomorphism (also denoted by $l$ ) from $\operatorname{sym}^{l}\left(V_{1}\right)$ to $\operatorname{sym}^{l}\left(V_{2}\right)$. For $j=1,2$, let $\rho_{j}$ be the representation $\operatorname{det}^{k} \otimes \operatorname{sym}^{l}$ of $G L(n, C)$ on $\operatorname{sym}^{l}\left(V_{j}\right)$ as in Sect. 2.

For $s \in C$ and $\lambda \in Z_{\geq 0}$, we put

$$
(s)_{\lambda}:= \begin{cases}s(s+1) \cdots(s+\lambda-1), & \text { if } \lambda>0, \\ 1, & \text { if } \lambda=0 .\end{cases}
$$

For $M=\left(\begin{array}{ll}A & B \\ C & D\end{array}\right) \in S p(n, \boldsymbol{R})$, we put

$$
M^{\uparrow}:=\left(\begin{array}{cccc}
A & 0 & B & 0 \\
0 & 1_{n} & 0 & 0 \\
C & 0 & D & 0 \\
0 & 0 & 0 & 1_{n}
\end{array}\right), \quad M^{\downarrow}:=\left(\begin{array}{cccc}
1_{n} & 0 & 0 & 0 \\
0 & A & 0 & B \\
0 & 0 & 1_{n} & 0 \\
0 & C & 0 & D
\end{array}\right) .
$$

For $k \in 2 Z_{>0}, s \in C$ and $Z \in \mathfrak{H}_{n}$, we define the Eisenstein series by

$$
G_{k}^{(n)}(Z, s):=\sum_{M \in P_{n, 0} \backslash \Gamma^{n}} j(M, Z)^{-k}|j(M, Z)|^{-2 s}
$$

where

$$
P_{n, r}:=\left\{\left(\begin{array}{cc}
* & * \\
0^{(n+r, n-r)} & *
\end{array}\right) \in \Gamma^{n}\right\}
$$

The right-hand side of (3.1) converges absolutely and locally uniformly for $k+2 \operatorname{Re}(s)>n+1$. We consider also

$$
E_{k}^{(n)}(Z, s):=\operatorname{det}(\operatorname{Im}(Z))^{s} G_{k}^{(n)}(Z, s) .
$$

As is well known from the Langlands theory, the Eisenstein series $E_{k}^{(n)}(Z, s)$ has a meromorphic continuation to the whole $s$-plane. Moreover by [11], $E_{k}^{(n)}(Z, s)$ is holomorphic in $s$ at $s=0$ for each $Z \in \mathfrak{H}_{n}$. So we define $E_{k}^{(n)}(Z):=E_{k}^{(n)}(Z, 0)$.

For $v \in \boldsymbol{Z}_{\geq 0}$ and $\lambda \in \boldsymbol{C}-\{j / 2 \mid j \in \boldsymbol{Z}, n-2 v+2 \leq j \leq 2 n-1\}$, let $\tilde{\mathscr{D}}_{\lambda}^{v}$ be the differential operator defined in [2], which acts on $C^{\infty}\left(\mathfrak{H}_{2 n}, \boldsymbol{C}\right)$. For $k, l \in \boldsymbol{Z}_{\geq 0}$, let $L^{k, l}$ be the differential operator defined in [3], which maps each element of $C^{\infty}\left(\mathfrak{H}_{2 n}, C\right)$ to in $C^{\infty}\left(\mathfrak{H}_{n} \times \mathfrak{H}_{n}, \operatorname{sym}^{2 l}\left(V_{1} \oplus V_{2}\right)\right)$.

Let $k, l, v \in 2 Z_{\geq 0}, \quad k-v>0, \quad s \in C$ and $k-v+2 \operatorname{Re}(s)>2 n+1$. For $Z, W \in \mathfrak{H}_{n}$, we put 


$$
F_{k, v, l}^{(n)}(Z, W, s):=\left(\mathscr{D}_{k, v, l, s} G_{k-v}^{(2 n)}\right)\left(\left(\begin{array}{cc}
Z & 0 \\
0 & W
\end{array}\right), s\right),
$$

where a differential operator $\mathscr{D}_{k, v, l, s}$ is defined by

$$
\mathscr{D}_{k, v, l, s}:=L^{k, l} \operatorname{det}(\operatorname{Im}(3))^{s} \tilde{\mathscr{D}}_{k-v+s}^{v} .
$$

Then $F_{k, v, l}^{(n)}(Z, W, s) \in M_{k, l}^{n}\left(\operatorname{sym}^{l}\left(V_{1}\right)\right)^{\infty} \otimes M_{k, l}^{n}\left(\operatorname{sym}^{l}\left(V_{2}\right)\right)^{\infty}$ and we have the following:

Proposition. We assume $v \neq 0$. Then we get

$$
\begin{aligned}
& F_{k, v, l}^{(n)}(Z, W, s) \\
& =\frac{\varrho_{k, v}^{(n)}(s)}{(2 \pi i)^{l}} \sum_{\mu=0}^{l / 2}\left(-\frac{1}{4}\right)^{\mu} a(l, \mu, k, s) \sum_{T \in \boldsymbol{T}^{(n)}} \mathscr{P}_{\mu}(Z, W, T, s) \operatorname{det}(T)^{v},
\end{aligned}
$$

where

$$
\begin{aligned}
& \varrho_{k, v}^{(n)}(s):=\prod_{\lambda=0}^{\nu-1} \prod_{j=1}^{n}\left(-(k-v+s)+\frac{j-1}{2}-\lambda\right), \\
& a(l, \mu, k, s):=\frac{1}{(k)_{l}} \sum_{j=\mu}^{l / 2}(-1)^{J-\mu}\left(\begin{array}{c}
j \\
\mu
\end{array}\right) \frac{(2 k-2+2 j)_{l-2 j}(-s)_{j}(k+s)_{l-j}}{j !(l-2 j) !(k-1+j)_{l-j}}, \\
& \boldsymbol{T}^{(n)}:=\left\{\left(\begin{array}{ccc}
t_{1} & & 0 \\
& \ddots & \\
0 & & t_{n}
\end{array}\right)\left|t_{j} \in Z_{>0}(j=1, \ldots, n), t_{1}\right| \cdots \mid t_{n}\right\}, \\
& \mathscr{P}_{\mu}(Z, W, T, s):=\sum_{\tilde{g}_{1} \in \Gamma^{n}} \sum_{\tilde{g}_{1}^{\prime} \in \Gamma^{n}(T) \backslash \Gamma^{n}}\left\{\operatorname{det}(\operatorname{Im}(Z))^{s} \operatorname{det}(\operatorname{Im}(W))^{s}\right. \\
& \times\left|\operatorname{det}\left(1_{n}-T W T Z\right)\right|^{-2 s} \rho_{1}\left(\left(1_{n}-T W T Z\right)^{-1}\right)\left(e_{1} T^{t} e_{2}\right)^{l-2 \mu} \\
& \times\left(e_{1}\left(1_{n}-T W T \bar{Z}\right) \operatorname{Im}(Z)^{-1 t}\left(1_{n}-T W T Z\right)^{t} e_{1}\right)^{\mu} \\
& \left.\times\left(e_{2}\left(1_{n}-T Z T W\right)^{-1}\left(1_{n}-T Z T \bar{W}\right) \operatorname{Im}(W)^{-1} t e_{2}\right)^{\mu}\right\}\left|\left(\tilde{g}_{1}^{\prime}\right)_{W}\right|\left(\tilde{g}_{1}\right)_{Z},
\end{aligned}
$$

where ()$_{Z}$ (resp. ()$\left._{W}\right)$ denotes the action on $Z$ (resp. $W$ ) and

$$
\Gamma^{n}(T):=\left\{g \in \Gamma^{n} \mid\left(\begin{array}{cc}
0 & T^{-1} \\
T & 0
\end{array}\right) g\left(\begin{array}{cc}
0 & T^{-1} \\
T & 0
\end{array}\right) \in \Gamma^{n}\right\} .
$$

Proof. For $3 \in \mathfrak{H}_{2 n}$,

$$
G_{k-v}^{(2 n)}(\mathbb{3}, s)=\sum_{\mathfrak{M} \in P_{2 n, 0} \backslash \Gamma^{2 n}} j(\mathfrak{M}, 3)^{-k+v}|j(\mathfrak{M}, 3)|^{-2 s} .
$$


By Garrett [4], the left coset $P_{2 n, 0} \backslash \Gamma^{2 n}$ has a complete system of representatives $g_{\tilde{T}} \tilde{g}_{1}^{\uparrow} g_{2}^{\uparrow} \tilde{g}_{1}^{\prime \downarrow} g_{2}^{\prime \downarrow}$ with

$$
\begin{aligned}
g_{\tilde{T}}= & \left(\begin{array}{cccc}
1_{n} & 0 & 0 & 0 \\
0 & 1_{n} & 0 & 0 \\
0 & \tilde{T} & 1_{n} & 0 \\
\tilde{T} & 0 & 0 & 1_{n}
\end{array}\right), \quad \tilde{T}=\left(\begin{array}{cc}
0 & 0 \\
0 & T^{(r)}
\end{array}\right), \quad T \in \boldsymbol{T}^{(r)} \quad(r=0, \ldots, n), \\
& \tilde{g}_{1} \in G_{n, r}, \quad g_{2} \in P_{n, r} \backslash \Gamma^{n}, \quad \tilde{g}_{1}^{\prime} \in \Gamma^{r}(T) \backslash G_{n, r}, \quad g_{2}^{\prime} \in P_{n, r} \backslash \Gamma^{n},
\end{aligned}
$$

where

$$
G_{n, r}:=\left\{\left(\begin{array}{cccc}
1_{n-r} & 0 & 0 & 0 \\
0 & A^{(r)} & 0 & B^{(r)} \\
0 & 0 & 1_{n-r} & 0 \\
0 & C^{(r)} & 0 & D^{(r)}
\end{array}\right) \in \Gamma^{n}\right\}, \quad \Gamma^{r}(T) \subset \Gamma^{r} \simeq G_{n, r}
$$

Hence we put $\mathfrak{M}=g_{\tilde{T}} \tilde{g}_{1}^{\uparrow} g_{2}^{\uparrow} \tilde{g}_{1}^{\prime} g_{2}^{\prime \downarrow}$.

By Böcherer [2, Lemma 10],

$$
\tilde{\mathscr{D}}_{k-v+s}^{v} j(\mathfrak{M}, 3)^{-k+v-s}=\varrho_{k, v}^{(n)}(s) \operatorname{det}(\tilde{T})^{v} j(\mathfrak{M}, 3)^{-k-s} .
$$

Therefore

$$
\begin{aligned}
& \operatorname{det}(\operatorname{Im}(3))^{s} \tilde{\mathscr{D}}_{k-v+s}^{v}\left(j(\mathfrak{M}, 3)^{-k+v}|j(\mathfrak{M}, 3)|^{-2 s}\right) \\
& =\varrho_{k, v}^{(n)}(s) \operatorname{det}(\tilde{T})^{v} j(\mathfrak{M}, 3)^{-k}|j(\mathfrak{M}, 3)|^{-2 s} \operatorname{det}(\operatorname{Im}(3))^{s} .
\end{aligned}
$$

If $\operatorname{rank} \tilde{T} \neq n$, then (3.3) is equal to 0 . So we suppose $\operatorname{rank} \tilde{T}=n$, i.e. $\tilde{T}=T \in \boldsymbol{T}^{(n)}$. Then we can put $g_{2}=1_{2 n}$ and $g_{2}^{\prime}=1_{2 n}$. By Takayanagi [9, Lemma 1, Proposition 2], we get

$$
\begin{aligned}
\left.\mathscr{D}_{k, v, l, s}\left(j(\mathfrak{M}, \mathbf{3})^{-k+v}|j(\mathfrak{M}, \mathbf{3})|^{-2 s}\right)\right|_{3=3_{0}} \\
=\frac{\varrho_{k, v}^{(n)}(s)}{(2 \pi i)^{l}} \operatorname{det}(T)^{v} \sum_{\mu=0}^{l / 2}\left(-\frac{1}{4}\right)^{\mu} a(l, \mu, k, s)\left\{\operatorname{det}(\operatorname{Im}(Z))^{s} \operatorname{det}(\operatorname{Im}(W))^{s}\right. \\
\quad \times\left|\operatorname{det}\left(1_{n}-T W T Z\right)\right|^{-2 s} \rho_{1}\left(\left(1_{n}-T W T Z\right)^{-1}\right)\left(e_{1} T^{t} e_{2}\right)^{l-2 \mu} \\
\quad \times\left(e_{1}\left(1_{n}-T W T \bar{Z}\right) \operatorname{Im}(Z)^{-1 t}\left(1_{n}-T W T Z\right)^{t} e_{1}\right)^{\mu} \\
\left.\quad \times\left(e_{2}\left(1_{n}-T Z T W\right)^{-1}\left(1_{n}-T Z T \bar{W}\right) \operatorname{Im}(W)^{-1} e_{2}\right)^{\mu}\right\}\left|\left(\tilde{g}_{1}^{\prime}\right)_{W}\right|\left(\tilde{g}_{1}\right)_{Z},
\end{aligned}
$$

where $\boldsymbol{Z}_{0}=\left(\begin{array}{ll}Z & 0 \\ 0 & Q\end{array}\right)$.

Thus, we obtain (3.2). 
4. Special values of $L(s, f, \underline{\mathrm{St}})$

THEOREM. Let $k, l \in 2 Z_{\geq 0}$ and $k \geq 2 n+2$. Let $f \in S_{k, l}^{n}\left(\operatorname{sym}^{l}\left(V_{2}\right)\right)$ be an eigenform with the Fourier coefficients in $\operatorname{sym}^{l}\left(\left(V_{2}\right)_{Q(f)}\right)$. Let $m \in Z$ be such that

$$
1 \leq m \leq k-n \text { and } m \equiv n(\bmod 2) \text {. }
$$

We assume

$$
n \equiv 3(\bmod 4) \text { if } m=1
$$

Let

$$
A(f):=\frac{L(m, f, \underline{\mathrm{St}})}{\pi^{n k+l+m(n+1)-n(n+1) / 2}(f, f)} .
$$

Then we have

$$
A(f)^{\sigma}=A\left(f^{\sigma}\right) \text { for all } \sigma \in \operatorname{Aut}(\boldsymbol{C})
$$

In particular,

$$
A(f) \in \boldsymbol{Q}(f)
$$

Proof. Let $f \in S_{k, l}^{n}\left(\operatorname{sym}^{l}\left(V_{2}\right)\right)$ is an eigenform. Taking the inner product of $f$ and (3.2) in the variable $W$, we obtain the following in the same way as in [9]:

$$
\begin{aligned}
\left(f, F_{k, v, l}^{(n)}(-\bar{Z}, *, \bar{s})\right) & \\
= & \overline{\varrho_{k, v}^{(n)}(\bar{s})} \frac{1}{(k)_{l} l !} 2^{n(n+1-k-2 s)-l+l} i^{n k} \pi^{n(n+1) / 2-l} \prod_{j=1}^{n-1} \frac{\Gamma(2 k+2 s+2 j-2 n-1)}{\Gamma(2 k+2 s+j-n-2)} \\
& \times \frac{\Gamma(k+s+l / 2-1) \Gamma(k+s+l / 2-1 / 2) \Gamma(k+s-n) \Gamma(2 k+2 s+l-n-1)}{\Gamma(k+s) \Gamma(k+s-1 / 2) \Gamma(k+s-1) \Gamma(2 k+2 s+l-2)} \\
& \times \zeta(2 s+k-v)^{-1} \prod_{j=1}^{n} \zeta(4 s+2 k-2 v-2 j)^{-1} L(2 s+k-v-n, f, \underline{\mathrm{St}}) \\
& \times\left(l^{-1}(f)\right)(Z) .
\end{aligned}
$$

Here the convergence of the left-hand side follows from [7, Theorem 5.4]. Moreover the expression holds if $v=0$ in [9]. We note that $L(s, f, \underline{\mathrm{St}})$ is holomorphic at $m$. We put $s=0$ and $v=k-n-m$, which satisfies the required condition: $v \in 2 Z_{\geq 0}$ and $k-v>0$. Then

$$
(f, g(-\bar{Z}, *))=c(f)\left(l^{-1}(f)\right)(Z),
$$

where

$$
\begin{aligned}
g(Z, W) & :=\pi^{-n(k-n-m)} F_{k, k-n-m, l}^{(n)}(Z, W, 0) \\
& =\left(\pi^{-n(k-n-m)} L^{k, l} \tilde{\mathscr{D}}_{m+n}^{k-n-m} E_{m+n}^{(2 n)}\right)\left(\begin{array}{cc}
Z & 0 \\
0 & W
\end{array}\right),
\end{aligned}
$$




$$
\begin{aligned}
c(f):= & \varrho_{k, v}^{(n)}(0) \frac{1}{(k)_{l} l !} 2^{n(n+1-k)-l+1} i^{n k} \pi^{n(n+1) / 2-l-n(k-n-m)} \\
& \times \prod_{j=1}^{n-1} \frac{\Gamma(2 k+2 j-2 n-1)}{\Gamma(2 k+j-n-2)} \\
& \times \frac{\Gamma(k+l / 2-1) \Gamma(k+l / 2-1 / 2) \Gamma(k-n) \Gamma(2 k+l-n-1)}{\Gamma(k) \Gamma(k-1 / 2) \Gamma(k-1) \Gamma(2 k+l-2)} \\
& \times \zeta(m+n)^{-1} \prod_{j=1}^{n} \zeta(2 m+2 n-2 j)^{-1} L(m, f, \underline{\mathrm{St}}) .
\end{aligned}
$$

Since

$$
\begin{gathered}
\zeta(m+n)^{-1} \prod_{j=1}^{n} \zeta(2 m+2 n-2 j)^{-1} \in \pi^{-\left(m+2 m n+n^{2}\right)} \boldsymbol{Q}^{\times}, \\
c(f)=\frac{L(m, f, \underline{\mathrm{St}})}{\pi^{n k+l+m(n+1)-n(n+1) / 2}} \times(\text { rational }) .
\end{gathered}
$$

Therefore it suffices to show that

$$
\left(\frac{c(f)}{(f, f)}\right)^{\sigma}=\frac{c\left(f^{\sigma}\right)}{\left(f^{\sigma}, f^{\sigma}\right)} \quad \text { for all } \sigma \in \operatorname{Aut}(C) .
$$

We have a partial Fourier expansion of $g(Z, W)$ :

$$
g(Z, W)=\sum_{R \geq 0} \sum_{\xi \in X_{n}} g_{R, \xi}(W) \xi e^{2 \pi l \operatorname{trace}(R Z)},
$$

where $X_{n}:=\left\{\prod_{j=1}^{n} x_{j}^{\alpha_{j}} \mid \alpha_{j} \in Z_{\geq 0}, \sum_{J=1}^{n} \alpha_{j}=l\right\}$, which is an orthogonal basis of $\operatorname{sym}^{l}\left(V_{1}\right)$. By Weissauer [11], $E_{m+n}^{(2 n)}$ is a holomorphic modular form with rational Fourier coefficients. Since $\pi^{-n(k-n-m)} L^{k, l} \tilde{\mathscr{D}}_{m+n}^{k-n-m}$ preserves the rationality of Fourier coefficients, we have

$$
g_{R, \xi} \in M_{k, l}^{n}\left(\operatorname{sym}^{l}\left(V_{2}\right)\right)_{Q} .
$$
obtain

From (4.1), for any symmetric, semi-integral, semi-positive matrix $R$, we

$$
\left(f, g_{R, \xi}\right)=c(f) a_{R, \xi}\left(l^{-1}(f)\right) \text { for all } \xi \in X_{n},
$$

where $a_{R, \xi}(\cdot)$ is the $\xi$-component of the Fourier coefficient $a_{R}(\cdot)$. Let $h_{R, \xi}$ be the projection of $g_{R, \xi}$ to $S_{k, l}^{n}\left(\operatorname{sym}^{l}\left(V_{2}\right)\right)$. Then we obtain

$$
\left(f, h_{R, \xi}\right)=c(f) a_{R, \xi}\left(l^{-1}(f)\right) \text { for all } \xi \in X_{n} .
$$

We fix $R$ and $\xi$ such that $a_{R, \xi}\left(l^{-1}(f)\right) \neq 0$. 
Let $\lambda \in \widehat{\mathrm{T}_{C}}$ be an eigenvalue on $f$. Let $\sigma \in \operatorname{Aut}(\boldsymbol{C})$ and $N:=\operatorname{dim}_{C} S_{k, l}^{n}(\lambda)$. Since $f \in S_{k, l}^{n}(\lambda)_{Q(\lambda)}$, by Takei [10, Theorem 1],

$$
f^{\sigma} \in S_{k, l}^{n}\left(\lambda^{\sigma}\right) \boldsymbol{Q}\left(\lambda^{\sigma}\right)
$$

and there exists an orthogonal basis $\left\{f_{J}\right\}_{J=1}^{N}$ of $S_{k, l}^{n}(\lambda)$ such that

$$
f_{1}=f \quad \text { and } \quad f_{j} \in S_{k, l}^{n}(\lambda)_{Q(\lambda)} \quad(j=1, \ldots, N) .
$$

Let $h(\lambda)$ be the projection of $h_{R, \xi}$ to $S_{k, l}^{n}(\lambda)$. Since $h_{R, \xi} \in S_{k, l}^{n}\left(\operatorname{sym}^{l}\left(V_{2}\right)\right)_{Q}$,

$$
h(\lambda)^{\sigma}=h\left(\lambda^{\sigma}\right) \text {. }
$$

Writing $h(\lambda)=\sum_{j=1}^{N} \beta_{j} f_{j}$, we have

$$
c(f) a_{R, \xi}\left(l^{-1}(f)\right)=\beta_{1}(f, f) .
$$

On the other hand,

$$
c\left(f^{\sigma}\right) a_{R, \xi}\left(l^{-1}\left(f^{\sigma}\right)\right)=\beta_{1}^{\sigma}\left(f^{\sigma}, f^{\sigma}\right) .
$$

Therefore

$$
\left(\frac{c(f)}{(f, f)}\right)^{\sigma}=\frac{\beta_{1}^{\sigma}}{a_{R, \xi}\left(l^{-1}\left(f^{\sigma}\right)\right)}=\frac{c\left(f^{\sigma}\right)}{\left(f^{\sigma}, f^{\sigma}\right)} .
$$

Thus Theorem is proved.

Acknowledgements. The author would like to thank Professor S. Mizumoto for introducing the subject to the author and giving helpful guidance.

\section{REFERENCES}

[1] A. N. ANDrianov, The multıplicatıve arıthmetıc of Siegel modular forms, Russian Math. Surveys, 34 (1979), 75-148.

[2] S. BöCHERER, Über die Fourıer-Jacobı-Entwicklung Siegelscher Eisensteınreihen II, Math. Z., 189 (1985), 81-110.

[ 3 ] S. BÖCHERER, T. SATOH, AND T. YAMAZAKI, On the pullback of a differential operator and its application to vector valued Eisenstein series, Comment. Math. Unıv. St. Paul., 42 (1992), $1-22$.

[4] P. B. Garretr, Pullbacks of Eisenstein series, Automorphic Forms of Several Variables (I. Satake and Y Morıta eds.), Progr. Math., 46, Birkhäuser, 1984, 114-137.

[5] M. HARris, Special values of zeta functions attached to Siegel modular forms, Ann. Sc1. École Norm. Sup., 14 (1981), 77-120.

[6] S. Mizumoto, Poles and residues of standard $L$-functions attached to Siegel modular forms, Math. Ann., 289 (1991), 589-612.

[ 7 ] S. Mizumoto, Eisenstein series for Siegel modular groups, Math. Ann., 297 (1993), 581-625.

[8] J. STuRM, The critical values of zeta functions associated to the symplectic group, Duke Math. J., 48 (1981), 327-350.

[ 9 ] H. TaKayanagi, Vector valued Siegel modular forms and their $L$-functions; Application of a differential operator, Japan J. Math., 19 (1994), 251-297. 
[10] Y TAKeI, On algebraicity of vector valued Siegel modular forms, Kodal Math. J., 15 (1992), $445-457$

[11] R. Weissauer, Stabile Modulformen und Eisenstemnreihen, Lecture Notes in Math., 1219, Berlin, Springer, 1986.

Department of Mathematics

ToKyo Institute of TeChNOLOGY

OH-OKAYAMA, TOKYo, 152-8551, JAPAN 